\title{
Chronic Lymphocytic Leukemia in New Era
}

\author{
Rasha I. Ibrahim, M.D. \\ Internal Medicine (Clinical Hematology Unit) \\ Ain Shams University
}

\begin{abstract}
:-
Treatment of Chronic Lymphocytic Leukemia (CLL) is entering a new era. Although CLL remains incurable, over the past two decade there have been tremendous advances in understanding the pathophysiology of CLL and in the treatment of this disease. In the last 5 years, several new exciting drugs have entered the realm in hopes for a success in curing the disease. These drugs are falling into different classes such as Bruton tyrosine kinase (BTK) inhibitors, B cell CLL/Lymphoma-2 (BCL-2) inhibitors, CD20 Antibody, phosphatidyl inositol 3-kinase (PI3K) inhibitors, and spleen tyrosine kinase (Syk) inhibitors. Numerous studies have shown that these therapeutic agents have both clinical activity as initial therapy and as treatment of recurrent disease. This has led to greatly treatment goal shifting from symptom palliation to achieve complete remission (CR) and improve survival. Advances in our understanding by using of some prognostic factors that help to identify early high risky patients for progression (including molecular factors) have led us to the question whether there is still a role for a "watch and wait" approach in asymptomatic high-risk patients or whether they should be treated earlier in their disease course. Questions are still remain regarding; what is the optimal first-line of treatment, its timing and what is the beneficial role of maintenance therapy or stem cell transplantation in this disease? The established treatment algorithms for CLL are currently challenged by novel classes of drugs. Targeted drugs are replacing the often-debilitating chemotherapy regimens that have long been the standard of care in CLL. Novel strategies combining or sequencing new agents with established chemotherapy and chemo-immunotherapies combinations are likely to change the treatment of CLL in the near future.
\end{abstract}

\section{Introduction:-}

Chronic Lymphocytic Leukemia is a monoclonal disorder characterized by a progressive accumulation of functionally incompetent lymphocytes; its onset is usually insidious, and it may be discovered incidentally after a blood cell count is performed for another reason. ${ }^{1}$ Enlarged lymph nodes are the most common presenting symptom, but patients may present with a wide range of symptoms and signs. ${ }^{1}$ It considers the most common disorder of all leukemic forms that can found in adults in Western countries; the American Cancer Society estimates that 18,960 new cases of CLL will be diagnosed in the United States in 2016. ${ }^{2}$ Although the incidence of CLL in Western countries is similar to that of the United States; CLL is extremely rare in Asian countries, where it is estimated to comprise only $10 \%$ of 
all leukemias. ${ }^{3}$ The incidence of CLL is higher among whites than blacks; and it is higher in males than in females, with a ratio of $1.7: 1 .^{3}$

Chronic Lymphocytic Leukemia is a disease that primarily affects the elderly, with the median age of presentation being 72 years. ${ }^{3}$ As in the case of most malignancies, the exact cause of CLL is uncertain; CLL is an acquired disorder, and reports of truly familial cases are exceedingly rare. ${ }^{4} \mathrm{~A}$ meta-analysis of four genome-wide association studies that included 3100 cases of CLL found multiple risk loci. Several of those loci are in close proximity to genes involved in apoptosis, suggesting a plausible underlying biological mechanism. ${ }^{5}$

\section{Pathophysiology:-}

Chronic Lymphocytic Leukemia is characterized by the clonal proliferation and accumulation of mature, typically CD5-positive B cells within the blood, BM, lymph nodes, and spleen. The leukemic transformation is initiated by specific genomic alterations, in particular deletions on the long arm of chromosome 13 del (13q14). ${ }^{6}$ Studies have demonstrated that the proto-oncogene bcl2 is over expressed in Bcell CLL. ${ }^{7}$ The proto-oncogene bcl2 is a known suppressor of apoptosis (programmed cell death), resulting in a long life for the involved cells. Studies have shown that this upregulation in bcl2 is related to deletions of band $13 q 14$. Two genes, named miRNA15a and miRNA16-1, are located at $13 q 14$ and have been shown to encode not for proteins, but rather for a regulatory RNA called microRNA (miRNA) ${ }^{8}$ These miRNA genes belong to a family of highly conserved non coding genes throughout the genome whose transcripts inhibit gene expression by causing degradation of mRNA or by blocking transcription of mRNA. Deletions of miRNA15a and miRNA16-1 lead to over expression of bcl2 through loss of down regulating miRNAs. Genetic analyses have demonstrated deletion or down regulation of these miRNA genes in $70 \%$ of CLL cases. ${ }^{9}$

Additional aberrations of the long arm of chromosome 11 del (11q), of the short arm of chromosome 17del (17p) which is associated with loss of function of the tumor suppressor gene p53, and trisomy 12 seem to occur later in the course of the disease and predict a worse outcome. ${ }^{10}$

In addition, whole-genome sequencing has uncovered recurrent somatic gene mutations that occur in CLL cells in parallel to the above-mentioned structural genomic aberrations. Of these, mutations affecting the genes NOTCH1, MYD88, TP53, ATM, and SF3B1 seem to be more common and to have prognostic impact. ${ }^{11}$

It has become increasingly clear that survival of CLL cells is not a cellautonomous, genetically determined process. Instead, survival of CLL cells strictly depends on a permissive microenvironment composed of cellular components such as macrophages, T cells, or stromal follicular dendritic cells, which provide essential proteins (chemokines, cytokines and angiogenic factors) for activation of crucial survival and proliferative signaling pathways of transformed cells. ${ }^{12}$

In addition, stimulation of the B-Cell Receptor (BCR) also drives the activation of different tyrosine kinases, such as (BTK), (Syk), zeta-associated protein70(ZAP70), Src family Kinases (in particular Lyn kinase), and (PI3K), which stimulate malignant B-cell survival via activation of transcription factors such as NFKB. ${ }^{13}$ The importance of BCR signaling is underscored by the fact that different features of the BCR have been recognized as a prognostic marker in CLL. These pathways have recently gained in importance because they can be inhibited by 
specific small-molecule inhibitors that show clinical efficacy in lymphoid malignancies. ${ }^{1}$

How to treat patient with Chronic Lymphocytic Leukemia in 2016?

Heterogeneity in the clinical course of the disease is one of the hallmarks of CLL. The use of novel biological and genetic parameters allows separating some patients in the high-risk subgroups, with a median survival $<3$ years, from those with a very mild course with a median survival $>25$ years.

Wait and watch policy was the standards care for most of patients with CLL for decades but multiple new prognostic factors and better understanding of pathogenesis of CLL, improve our ability to predict the natural history of CLL and permit tailoring of treatment to individual patients.

So in 2016 before you decide about your plan of management of your patient with CLL you should answer some questions:-

- What is the prognosis?

- When should treatment start?

- What is the fitness of your patients and his comorbidities?

- What type of therapy should you choose?

- What is your goal of therapy?

Nowadays there is numerous clinical and molecular features are predictive of the course of CLL and can be used for risk stratification (Table1). ${ }^{14}$

Table (1):- Clinical and Novel Prognostic Factors in Chronic Lymphocytic Leukemia

\section{Adverse Clinical/Laboratory Prognostic Factors}

1. Advanced age

2. Advanced stage (Rai III/IV or Binet C)

3. Poor performance status

4. Short lymphocyte doubling time (<12 month)

5. Diffuse bone marrow infiltration pattern

6. Increased percentage of prolymphocytes

7. Male sex

8. High lactate dehydrogenase

9. High $\beta 2$-microglobulin and specifically if the levels are above $4 \mu \mathrm{g} / \mathrm{mL}$.

10-High serum levels of thymidine kinase, and TNF.

11. Increased levels of soluble CD23

\section{Novel/Molecular Adverse Prognostic Factors}

1. $17 p$ and $11 q$ deletions by fluorescence in situ hybridization

2. CD38 over expression ( $>30 \%$ )

3. Zap-70 greater than $20 \%$

4. Un-mutated IgVH

5. NOTCH-1 mutations

6. High lipoprotein lipase expression

7. Variance expressions of specific micro-RNAs

(ie, down-regulation of miR-15a and miR-16-1 is associated with good prognosis, 
The molecular profile of CLL provides insight into the underlying pathogenesis of the disease and provides predictors of time to progression, time to need for therapy, and overall survival. A molecular profile can be built from assessment of number of molecular biomarkers, the most important being cytogenetic analysis by fluorescence in situ hybridization (FISH), mutational status $(\operatorname{lgVH})^{15},(\text { ZAP70) })^{16}$, and CD38 expression ${ }^{17,18}$.

High-risk features predictive of disease progression include the cytogenetic features deletion of the long arm of chromosome 11 (del 11q) ${ }^{16}$ and del $17 p^{16}$, IgVH un-mutated status, and expression of ZAP70 and CD38.

-Del (17p13) results in the deletion of TP53, a tumor suppressor gene that is lost or mutated in a variety of malignancies. Loss of p53 leads to deregulated cell division. It is found in 5-7\% of CLL cases in early stages, while it

is present in $25-40 \%$ of patients with advanced refractory disease. Moreover, the presence of TP53 mutations, not always found along with the 17p deletion, is also of great relevance. The incidence of TP53 mutations in untreated patients is approximately $10 \%$ of the cases, whereas higher percentages are found in CLL refractory to fludarabine. CLL patients with deletion of TP53 are associated with poor prognosis with rapid progression of disease, poor response to therapy and short survival. ${ }^{16}$

The advent of next-generation sequencing technologies, coupled with gene copynumber analyses, have identified additional genetic lesions in CLL, such as mutations in NOTCH1, SF3B1, and BIRC3. Such mutations could be used as potential therapeutic targets or as prognostic markers that can distinguish among patients who may have disparate clinical outcomes. ${ }^{11}$

A current challenge is to understand how we should use this new information in clinical practice and whether we should alter our treatment based upon the detection of high-risk features. There is not yet any evidence that patients presenting with high-risk disease features have any benefit with earlier treatment and patients should not be offered treatment on the basis of any molecular marker until the standard criteria for treatment are reached.

What are indications to start therapy for Chronic Lymphocytic Leukemia? Most of patients seen in earlier stage of the disease so it is recommended to follow newly diagnosed patients who is asymptomatic with early-stage disease (Rai 0 , Binet A) every 3 months for history, physical examination, and blood counts without initiation of any therapy. This allows assessment of disease progression and measurement of the lymphocyte doubling time. Once it is established that patients are following a particularly stable clinical course, less frequent follow-up is sufficient if he has good molecular prognostic factors.

In all other leukemia as early treatment is optimal, but this is not the case in CLL as it has indolent course. Previous trials have demonstrated no survival advantage of early treatment versus an initial watch and wait approach (It should be noted that all these studies were performed using alkylating agents). ${ }^{19}$

We should start treatment if there is any evidence of disease progression ${ }^{20}$ as following:-

1. Evidence of progressive bone marrow failure as evidenced by anemia, thrombocytopenia, or both.

2. Massive ( $>6 \mathrm{~cm}$ below the left costal margin), symptomatic, or progressive splenomegaly. 
3. Massive $(>10 \mathrm{~cm})$ adenopathy or progressive symptomatic adenopathy.

4. Progressive lymphocytosis: $>50 \%$ increase in 2 months or lymphocyte doubling time less than 6 month.

5. Autoimmune anemia or thrombocytopenia not responsive to standard therapies.

6. Constitutional symptoms: unintentional weight loss greater than $10 \%$ over the preceding 6 months, unexplained night sweats for more than 1 month, unexplained fevers $>38.1^{\circ} \mathrm{C}$ for2 weeks.

What type of therapy should you choose?

First we should know that CLL is not currently curable disease by conventional chemotherapy or antibody-based therapies. The only curative option is allogeneic stem cell transplantation (HSCT) but as most of CLL patients are old age and have multiple comorbidities HSCT is not the best option for them as it has a risk of morbidities and mortalities so it is used in certain circumstances as we will discuss later. The choice of treatment for CLL depends on the fitness of your patient, the genetic status and patient age.

So how can you judge on your patient fitness?

This depends on presence of any comorbidities (determined e.g. by the CIRS / Cumulative Illness Rating Scale score), renal function and ECOG performance status. ${ }^{21}$ We consider patient is fit if CIRS score $<6$ and creatinine clearance $>60$ $70 \mathrm{ml} / \mathrm{min}$. Unfit patient has renal function impairment and increased comorbidities as CIRS score $>6$.Frail patient has a very poor general condition. These patients should receive only supportive measurements and this may also include administration of antineoplastic drugs such as steroids, chlorambucil, bendamustine or rituximab at adapted doses.

What are different drugs used in therapy for CLL?

Historically, CLL was treated with alkylating agent and purine analogue chemotherapies. Recently, the addition of anti CD-20 immunotherapy and B-Cell Receptor Pathway and Tyrosine Kinase Inhibitors to chemotherapeutic treatment of CLL has improved response and survival (Table 3). ${ }^{22}$

Table (3): Categories of Drug Therapy for Chronic Lymphocytic Leukemia.

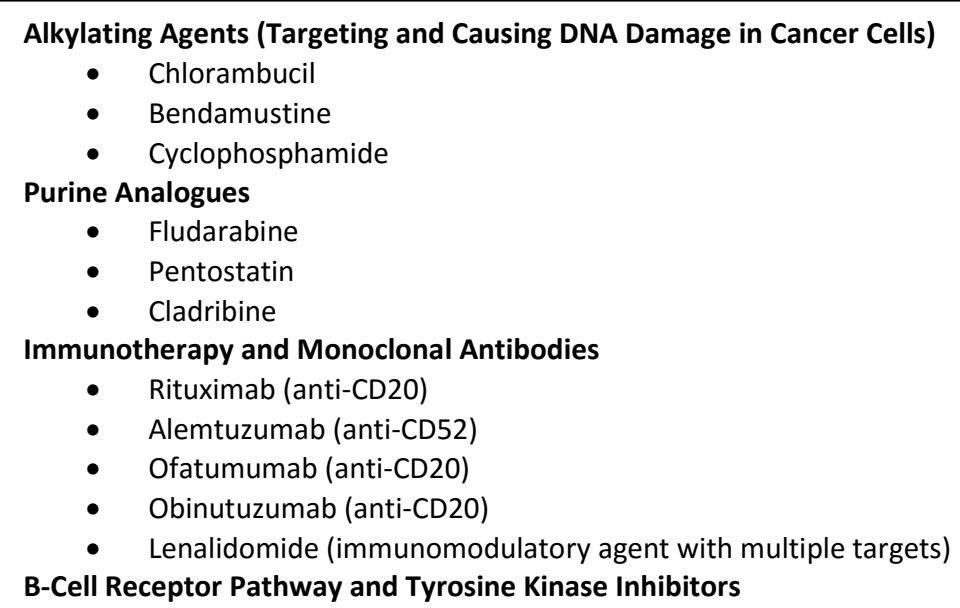


Treatment of CLL in the past was very simple approach as shown in (Figure1)

Figure 1:Treatment approach of Chronic Lymphocytic Leukemia in past.

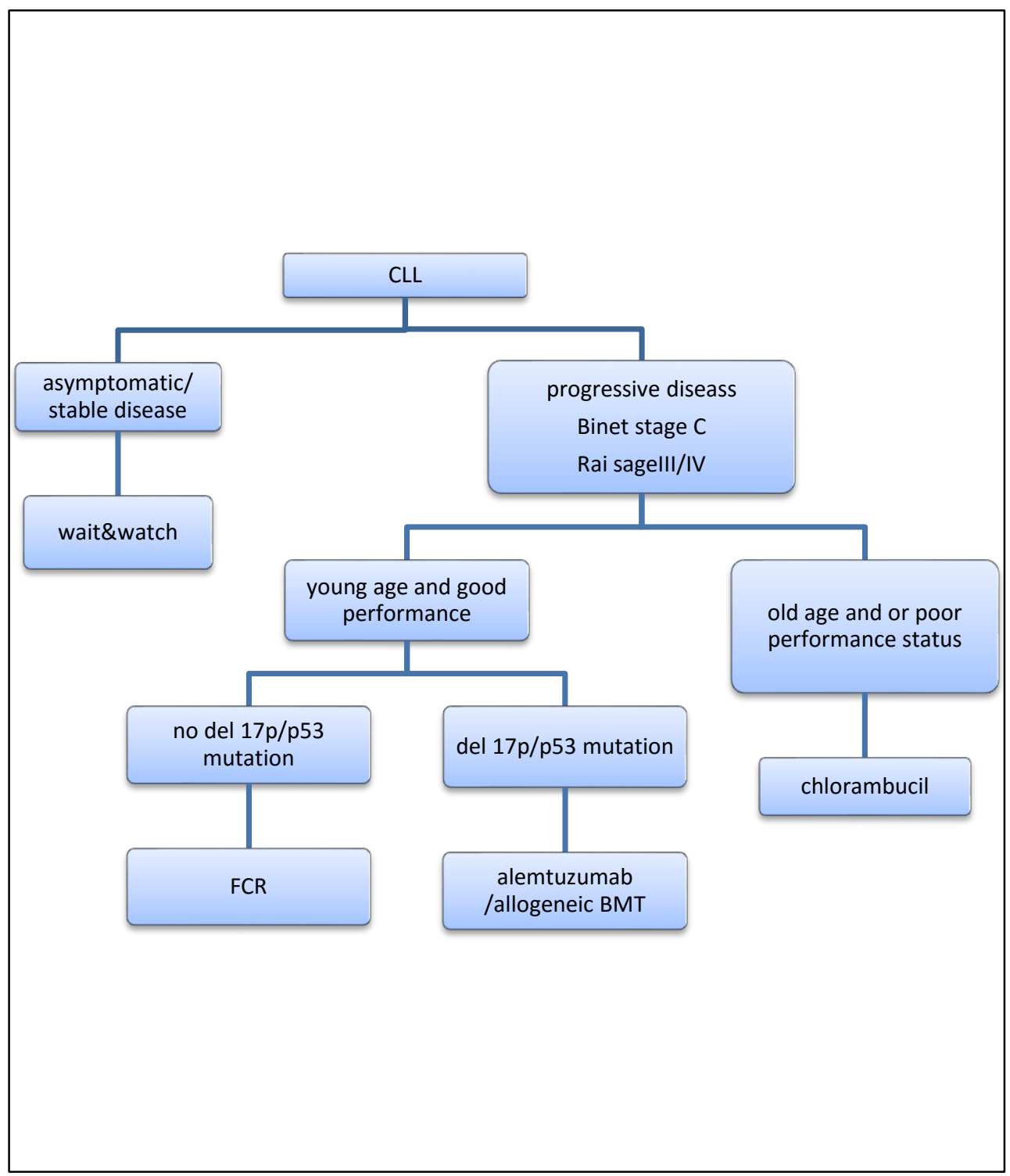




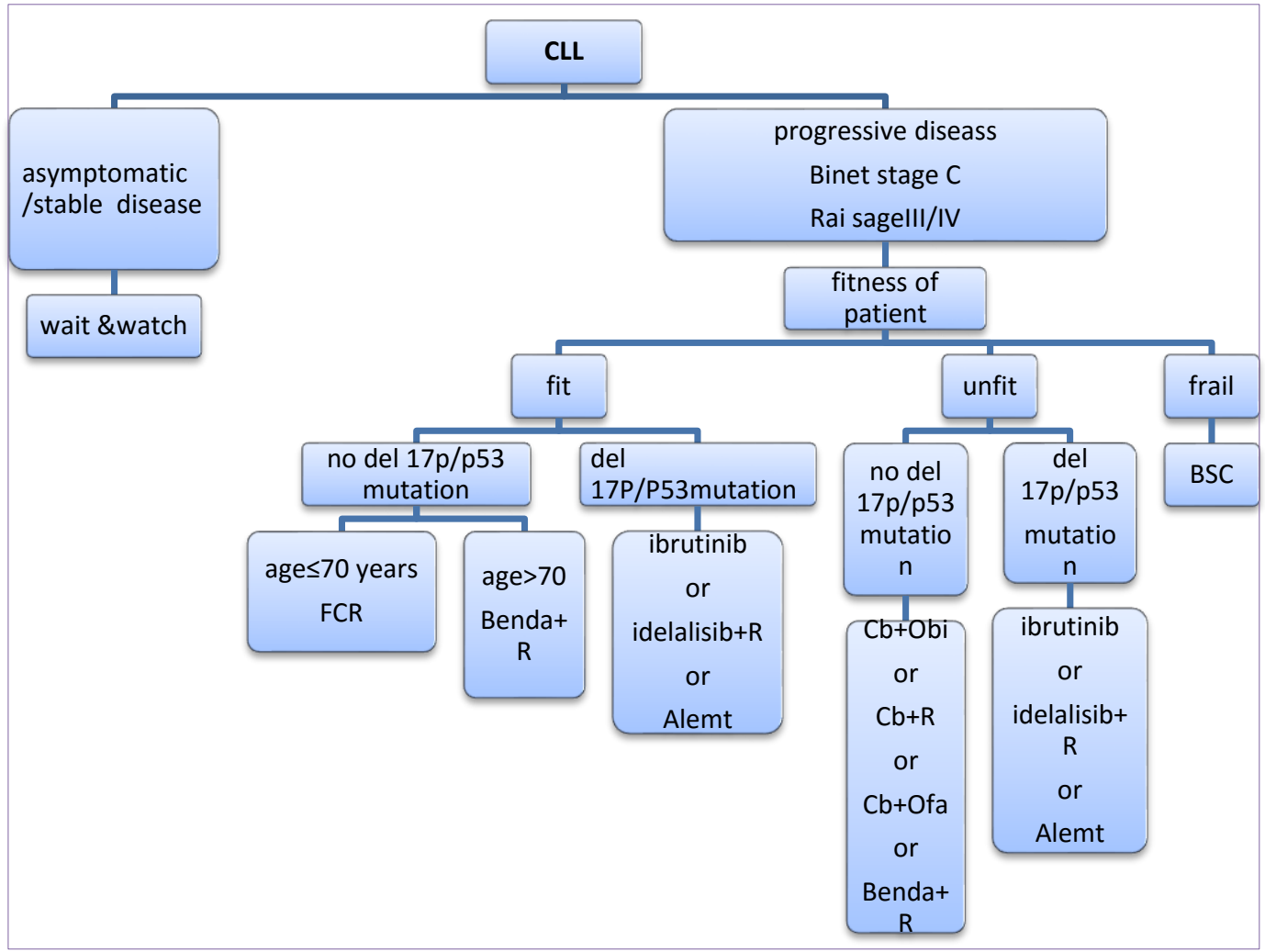

But in new era the treatment plan is changed dramatically by adding new targeted therapy as shown in (Figure2)

Figure 2: Treatment approach of Chronic Lymphocytic Leukemia in new era.Abbreviation :- Alemt - alemtuzumab, Benda - bendamustine, BSC Best Supportive Care, C - cyclophosphamide, Cb - chlorambucil, F fludarabine, , Obi - obinutuzumab, Ofa - Ofatumumab, , R - Rituximab;

What is our goal of therapy in Chronic Lymphocytic Leukemia?

In the past our aim is to achieve complete remission (CR),Cri or partial remission (PR).Now the complete eradication of the leukemia is an obvious desired endpoint. New detection technologies, such as multicolor flowcytometry and real-time quantitative PCR are used nowadays to detect minimal residual disease (MRD). it is reliably sensitive down to a level of approximately one CLL cell in10 000 leukocytes. As such, patients will be defined as having a clinical remission in the absence of MRD when they have blood or marrow with less than one CLL cell per 10000 leukocytes. This test should be done at the period within3 months of completing therapy. ${ }^{23}$ Therefore, future clinical trials that aim toward achieving long-lasting CR should include at least one test to assess MRD because the lack 
of leukemia persistence using these sensitive tests seems to have a strong, positive prognostic impact.

(FCR Protocol still is the standard protocol for fit patient with CLL)

As seen in our algorithm if patient is fit he should be treated with standard protocol combination of fludarabine and cyclophosphamide with rituximab (FCR).

The phase III GCLLSG CLL8 trial compared treatment with FCR or FC in younger fit CLL patients. ${ }^{24}$ The primary end point was progression-free survival (PFS). With a median follow-up of 5.9 years, median PFS were 56.8 and 32.9 months for the FCR and FC group $(P<.001)$. Median overall survival $(O S)$ was not reached for the FCR group and was 86.0 months for the FC group $(P=.001)$. In patients with mutated IGHV, FCR improved PFS and OS compared with FC (PFS: $P<.001$; OS:, $P=.1$ ). This improvement remained applicable for all cytogenetic subgroups other than del(17p). Long-term safety analyses showed that FCR had a higher rate of prolonged neutropenia during the first year after treatment $(16.6 \%$ vs $8.8 \% ; P=$ .007). Secondary malignancies including Richter's transformation occurred in $13.1 \%$ in the FCR group and in $17.4 \%$ in the FC group $(P=.1)$. First-line chemoimmunotherapy with FCR induces long-term remissions and highly relevant improvement in OS. Based on the results from the CLL-8 trial, FCR is currently the best option for the first-line treatment of young, fit patients with CLL. ${ }^{25}$

(Bendamustin +rituximab is less toxic protocol for elder patient with CLL) the phase III GCLLSG CLL10 trial randomized 564 physically fit CLL patients with low comorbidity burden to FCR or BR, with a median follow-up of 35.9 months. ${ }^{26}$ The overall response rate in both arms was the same $97.8 \%(p=1.0)$. Complete response rate was $40.1 \%$ in the FCR arm compared to $38.1 \%$ in the BR arm $(p=0.03)$. Amongst 355 evaluable patients, $74.1 \%$ and $62.9 \%$ for FCR and BR, respectively, tested negative for minimal residual disease $(p=0.024)$ (based on peripheral blood). Bone marrow biopsy at 12 months showed $58.2 \%$ of FCR patients compared to $26.3 \%$ of BR patients were MRD negative $(p<0.001)$. Median progression-free survival was 53.7 months in the FCR arm compared to 43.2 months in the BR arm ( $p=0.001)$, however, no statistically significant differences in OS were reported $(p=0.910)$. A subgroup analysis revealed no difference in PFS in patients over 65 years of age. Severe neutropenia was observed more frequently in the FCR arm ( $87.7 \%$ vs $67.8 \%$; $p<0.001)$, however, no significant differences were reported for rates of anemia $(p=0.46)$ or thrombocytopenia $(p=0.096)$. Severe infections occurred significantly more frequently in the FCR arm (39.8\% vs $24.4 \% ; p=0.001)$ during treatment phase until 6 -months follow-up, especially in older patients $(48.4 \%$ vs $26.8 \%$; $p=0.001)$. Treatment related mortality was $3.9 \%$ in the FCR arm and $2.1 \%$ in the BR arm. ${ }^{26}$

Based on these results, FCR should still be considered the standard of care for those who are young and physically fit. However, based on the CLL10 study results showing no improvement in OS or PFS in patients aged $\geq 65$ years and substantial grade 3 and higher hematologic toxicity and infection in such patients receiving FCR, we favor BR in this patient population.

Which protocol will you choose for treatment of elder frail patients with Chronic Lymphocytic Leukemia: Chlorambucil-rituximab or Chlorambucilobinutuzumab (GA101)?

Our Goal in treatment of elderly unfit patients with CLL is to: prolong life but balance efficacy and tolerability so the optimal frontline therapy for elderly patients with CLL and comorbidities was recently evaluated in a phase 3 randomized 
clinical trial of chemoimmunotherapy(CLL11 protocol of the GCLLSG) comparing chlorambucil (Clb) plus obinutuzumab [GA-101] (GClb) with Clb plus rituximab (RClb) versus Clbmonotherapy. ${ }^{27}$ The median age of the population was73, CIRS score was 8 and median GFR was $63 \mathrm{~mL} / \mathrm{min}$. Both antibody containing groups (GClb and $\mathrm{RClb}$ ) proved significantly better than Clb monotherapy in terms of ORR and PFS. GClb was associated with improved PFS compared to RClb (26.7 months versus 15.2 months, $p<0.0001)$. GClb also conferred an overall survival benefit over Clbmonotherapy $(p=0.002)$. Minimal residual disease (MRD) negativity, which in the CLL8 trial was shown to correlate with improved PFS and OS, was observed significantly more frequently with GClb than RClb. Toxicities were similar with the chemoimmunotherapy groups with an increase in infusionrelated reactions (IRRs) in the GClb-treated patients, with IRRs typically occurring only with Cycle 1 . There was no difference in severe infections or treatment related deaths between the three groups. An update of this trial also reported an improvement in OS in the patients in the $\mathrm{RClb}$ group compared to $\mathrm{Clb}$ monotherapy. In patients who are frail or elderly, the addition of an anti-CD20 monoclonal antibody (rituximab or GA101) results in improved PFS and OS over Clb monotherapy. The use of the novel monoclonal anti-CD20 antibody obinutuzumab (GA 101) improves PFS over rituximab and a larger improvement in OS over Clb monotherapy.

What are different CD20 monoclonal antibodies used in CLL and how it is effective?

\section{1-Rituximab:}

Rituximab is a chimeric anti-CD20 monoclonal antibody with anti-leukemia action, including complement-dependent lysis (CDC), antibody-dependent cell-mediated cytotoxicity (ADCC), and direct induction of apoptosis. ${ }^{28}$ Rituximab when given as a single agent has more limited clinical activity in CLL than in follicular lymphoma, unless very high doses are used. ${ }^{29}$ In contrast, combinations of rituximab with chemotherapy have proven to be very efficacious therapies for CLL as in FCR and BR protocols.

\section{2-Ofatumumab}

Ofatumumab is a fully humanized antibody targeting a unique epitope on the CD20 molecule expressed on human B cells, resulting in increased binding affinity to CD20, prolonged dissociation rate, and increased cell kill due to greater complement dependent cytotoxicity and similar antibody-dependent cellular cytotoxicity activity compared with rituximab, especially in cells expressing low levels of CD20. Ofatumumab has shown some efficacy in patients who are fludarabine and alemtuzumab refractory or who have bulky disease $(>5 \mathrm{~cm}) .{ }^{30}$ The ORR was $58 \%$ in the fludarabine and alemtuzumab (FA)-refractory group and $47 \%$ in the bulky disease group. A recently concluded randomized phase III study complement 1 (OMB110911) trial demonstrated increased efficacy of Ofatumumab in combination with chlorambucil in front-line treatment of CLL patients who are poor candidates for fludarabine-based therapy. Furthermore, results compared favorably with those of the combination of chlorambucil and rituximab, side effects were manageable, and treatment was generally well tolerated.

\section{3-Obinutuzumab:}

Obinutuzumab (GA101) is a unique, glycol-engineered type II anti-CD20 monoclonal antibody that showed impressive results in vitro with higher rates of apoptosis in malignant $\mathrm{B}$ cells compared with rituximab. ${ }^{31}$ The humanization of the 
parental B-Ly1 mouse antibody and subsequent glycol -engineering lead to higheraffinity binding to the CD20 type II epitope, increased antibody-dependent cellular cytotoxicity, low complement-dependent cytotoxicity activity, and increased direct cell death induction. ${ }^{32}$

Obinutuzumab may provide an advantage when combined with chemotherapy as discussed before in (CLL11 protocol of the GCLLSG).Major side effects included infusion reaction in $20 \%$ infections, Neutropenia, thrombocytopenia (10\%), and tumor lysis syndrome, all of which resolved. There was no dose-limiting toxicities. ${ }^{27}$

\section{Is still a role of Alemtuzumab in treatment of CLL?}

Alemtuzumab is a recombinant, fully humanized monoclonal antibody against the CD52 antigen. Alemtuzumab acts via a p53 independent mechanism, and has improved results in patients with $\operatorname{del}(17 p)$ compared to conventional chemotherapies. ${ }^{33}$

Evidence of the role of alemtuzumab in high-risk patients was first shown in the refractory setting in a study by Stilgenbauer et al., who reported a response rate of $54 \%$ in fludarabine-refractory patients with del(17p) or p53 abnormalities. ${ }^{34}$

A single arm, open-label study of patients with CLL with del(17p) also examined alemtuzumab in combination with high dose steroids, $82 \%$ percent of patients had an objective response with $36 \%$ achieving a CR. The median PFS and OS of previously untreated patients were 18.3 months and 38.9 months, respectively, improved compared to all historical controls for this patient population. ${ }^{35}$

Also it has a role in refractory cases; the combination of fludarabine and alemtuzumab (FluCam) was compared in a Phase III study to fludarabine monotherapy in 335 patients with relapsed/refractory CLL after 1 prior line of therapy. Patients receiving FluCam had a significantly improved PFS and had a significantly improved OS. Adverse events were similar in the 2 groups with the exception of cytomegalovirus events (14\% in the FluCam group compared to $<1 \%$ in the fludarabine monotherapy group) and an increase in infusion-related reactions, which were generally mild (Grade 1 or 2 ). This regimen will rarely be required in the era of novel agents because of the reduced access to alemtuzumab and the infectious risks and CMV monitoring requirements. ${ }^{36}$

How Targeting B-cell receptor signaling is changing the paradigm for CLL treatment?

Treatment for CLL is undergoing a dramatic shift: Targeted drugs are replacing the often-debilitating chemotherapy regimens that have long been the standard of care in CLL.

Recognition (BCR) signaling is essential for the proliferation and survival of (CLL) cells stands as one of the most important insights into the pathobiology of the disease as it is markedly up regulated in B-cell malignancies. Accumulated evidence supports that antigen dependent and -independent BCR signaling plays a central role in the pathogenesis of CLL. ${ }^{37}$

Well-characterized molecular markers correlated with adverse prognosis are now understood to be associated with and/or potentiate BCR-signaling activity, likely accounting for the more rapid progression of disease in cases where these features are present. ${ }^{38}$

More recently, kinases immediately downstream of the BCR, including (SYK) and (PI3K), have been found to be constitutively activated in the majority of CLL patients. These kinases and downstream amplification kinases such as (BTK) appear essential not only for activation of multiple survival pathways (Akt, Erk, 
nuclear factor $\mathrm{kB}$ ) but also for chemokine mediated migration and adhesion of $\mathrm{B}$ cells in the microenvironment. ${ }^{39}$

Ibrutinib:

An orally bioavailable small molecule, irreversibly inactivates BTK through covalent binding of a cysteine residue (Cys481) near the active site .BTK is a cytoplasmic tyrosine kinase of the TEC family that is essential for BCR signaling, NF-kB activation, and cellular proliferation. Owing to its covalent binding to BTK, ibrutinib can be dosed once daily, despite a short half-life. ${ }^{40}$

Ibrutinib is generally well tolerated. The most commonly reported side effects ( $\geq 20 \%$ of patients on single agent), are mostly grade 1-2 and transient, and include diarrhea, nausea, vomiting, dyspepsia, hypertension, upper respiratory tract infections, urinary tract infections, fatigue, cough, arthralgia, muscle cramps, nail ridging, bruising, rash, pyrexia, and peripheral edema. ${ }^{41}$ an early increase in lymphocytosis is typically noted by day 7 and persists for 2 to 3 months before slowly declining over time, concomitant with notable reduction in lymph node and spleen size and improvement in cytopenias this may be explained by its ability to induce a "compartmental shift" of malignant lymphocytes out of the BM andlymph nodes and into the peripheral blood. ${ }^{42}$

This drug is currently FDA approved for patients with relapsed or refractory CLL and for patients with CLL with del (17p).

First it was indicated for refractory/relapsed CLL as single agent as shown in an open label phase III study (RESONATE), 391patients with relapsed or refractory CLL or SLL were randomlyassigned to receive daily ibrutinib or the antiCD20mAbofatumumab. The ORR was superior in ibrutinib arm $(42.6 \%$ in the ibrutinib arm and $4.1 \%$ in the ofatumumab arm). An additional $20 \%$ of ibrutinibtreated patients had PR. At six months, the PFS with ibrutinib is also superior (it was $88 \%$ compared to $65 \%$ with ofatumumab). Ibrutinib also significantly improved OS at 12 months $(90 \%$ vs. $81 \%) .{ }^{43}$

Now it is indicated for the treatment of patients with chronic lymphocytic leukemia (CLL) with $17 p$ deletion. Farooqui and colleagues enrolled 51 patients with del(17p) or TP53 mutations; 35 were previously untreated and 16had relapsed/refractory CLL. The ORR at 24 weeks of treatment was $92 \%$. The estimated PFS for all patients at 24 months was $82 \%$. Five patients (10\%) progressed, 3 with Richter's transformation and 2withprolymphocytic transformation. The cumulative incidence of progression was $20 \%$ for relapsed/refractory CLL, $9 \%$ in previously untreated patients. ${ }^{44}$

Some ongoing trails are discussing using ibrutinib in combination with other drugs as following:

The combination of ibrutinib with rituximab for 6 cycles followed by ibrutinib until disease progression was well tolerated and resulted in an ORR of $95 \%$ with $8 \%$ CRs in patient with high-risk disease features. The estimated PFS at 18 months in all patients was $78 \%$ and was $72 \%$ in patients with del(17p) or TP53 mutation. ${ }^{45}$ The combination of ibrutinib $420 \mathrm{mg}$ with ofatumumab was explored using 3 different administration sequences; ibrutinib lead-in, concurrent start, or ofatumumab lead-in. Both ORR at $100 \%$ and 12 -month PFS at $89 \%$ was best in the ibrutinib lead-in cohort. ${ }^{46}$

In a phase $1 \mathrm{~b}$ study, 30 patients with relapsed/refractory CLL received ibrutinib with bendamustine and rituximab (BR). BR was given for up to 6 cycles and ibrutinib at $420 \mathrm{mg}$ was given continuously from day 1 onwards. No added toxicities were observed beyond what would be expected with BR alone. At a median follow 
up of 15.8 months, the ORR and CR rates were $93 \%$ and $17 \%$.Responding patients continued ibrutinib on an extension study, increasing the median follow up to 37.3 months and the rate of CRs to $40 \%$. The median time to CR was 18.2 months. The estimated PFS was $86.3 \%$ at 12 months $78.6 \%$ at 24 months, and $70.3 \%$ at 36 months. ${ }^{47}$

\section{Idelalisib}

The oncogenic PI3K/AKT pathway has been well characterized and is critical for essential cellular processes such as metabolism, growth, and proliferation. Activation leads to inhibition of apoptotic signals while concurrently increasing survival signals through activation of NF-KB. ${ }^{48}$ Although PI3K is broadly expressed in multiple cell types, the p110 delta and p110 gamma isoforms are expressed primarily in cells of hematopoietic origin and therefore represent a promising therapeutic target for lymphoma therapy. Inhibition of these isoforms has also been shown to affect the microenvironment through suppression of tumor-associated inflammation, cell signaling, and angiogenesis. ${ }^{49}$

Idelalisib is an orally bioavailable, selective, and reversible inhibitor of the PI3K isoform. ${ }^{50}$ It is indicated in relapsed CLL, specifically for its use in combination with rituximab in patients for whom rituximab alone would be considered appropriate therapy due to other existing medical conditions (comorbidities).with great benefit for high risk patients.

idelalisib combined with rituximab was compared to rituximab with placebo in 220 frail patients with relapsed CLL. The ORR (allPRs) was $81 \%$ in the idelalisib group, as compared with $13 \%$ in the placebo group. At 24 weeks, the PFS was $93 \%$ in the idelalisib group and $46 \%$ in the placebo group. The benefit of idelalisib and rituximab was similar in groups stratified by status of del(17p), presence of TP53 mutation, and IGHV mutation status. ${ }^{51}$

Idelalisib was evaluated as initial therapy for CLL in frail patients, 64 treatmentnaïve older patients with CLL or small lymphocytic leukemia (median age, 71 years) were treated with rituximab $375 \mathrm{mg} / \mathrm{m}^{2}$ weekly $\times 8$ and idelalisib $150 \mathrm{mg}$ twice daily continuously for 48 weeks. Patients completing 48 weeks without progression could continue to receive idelalisib on an extension study. The median time on treatment was 22.4 months. The overall response rate (ORR) was $97 \%$, including $19 \%$ CR. The ORR was $100 \%$ in patients with del(17p)/TP53 mutations and $97 \%$ in those with unmutated IGHV. Progression-free survival was $83 \%$ at 36 months. ${ }^{52}$

Idelalisib prescribing information contains a black box warning for fatal and/or severe diarrhea or colitis, hepatotoxicity, pneumonitis, and intestinal perforation. ${ }^{53}$ Diarrhea was one of the most common side effects that led to idelalisib dose reduction and treatment discontinuation. Two types of diarrhea are observed with idelalisib. The first type tends to be mild, self-limiting and generally occurs within the first eight weeks; the second type tends to occur relatively late, responds poorly to antidiarrheal or antimicrobial therapy, and is considered to be idelalisib-related .Endoscopies in patients with treatment-emergent, late onset diarrhea revealed the histological features overlapped with those found in autoimmune enterocolitis, graft-versus-host disease (GvHD), and cytomegalovirus (CMV) colitis. ${ }^{54}$

\section{Other BCR inhibitors:}

-Fostamatinib, which inhibits SYK and dozens of other kinases, was the first BCR inhibitor to be studied in patients with relapsed/refractory NHL and CLL. The dose 
limiting toxicity was a combination of diarrhea, neutropenia, and thrombocytopenia. The ORR in 11 patients with CLL was $55 \% .{ }^{55}$

In the eight years since the first patient received the SYK inhibitor fostamatinib, there has been a rapid clinical development of multiple BCR inhibitors. In addition to ibrutinib, at least three other covalent BTK inhibitors have entered clinical trials (CC-292, ONO-4059, and ACP-196).In addition, several noncovalent BTK inhibitors are in preclinical or very early clinical development .Additional PI3Kס inhibitors in clinical testing include duvelisib (formerly IPI-145, also inhibits PI3Ky), TGR-1202, and AMG-319. ${ }^{56}$

BCR inhibitor carry the effect of High ORR however ; deep remissions infrequent., it show a durable responses with continued treatment despite the presence of residual disease it is well tolerated oral agents associated with rapid improvement in lymphadenopathy, cytopenias, and disease symptoms with limited myelosuppression. It is effective in high-risk disease including in patients with del(17p).BCR inhibitors are clearly among the most effective agents developed for the treatment of CLL and could become the backbone of hopefully curative combination therapy.

What do you know about Resistance to BCR inhibitors and disease transformation?

Consistent observation in studies with BCR inhibitors has been that patients with del(17p) or del(11q), bulky disease, or unmutated IGHV respond as well as patients without these adverse disease features. ${ }^{43,51}$ With longer follow up, it was observed that disease progression occurred more often in patients with high-risk genetic features. ${ }^{44,57}$ In some patients, disease progression manifests as large $B$ cell lymphoma (Richter's transformation).Progression with Richter's transformation tends to occur within the first 12-18 months on treatment, while progression with CLL tends to occur later. ${ }^{58}$

Mechanisms of resistance differ depending on which kinase is targeted.

- Recently, acquired mutations in BTK(e.g.a cysteine-to-serine mutation in $\mathrm{BTK}(\mathrm{C} 481 \mathrm{~S})$ at the binding site of ibrutinib that prevents the covalent binding of the drug and thereby dramatically decreases the potency of ibrutinib and in phospholipase C, gamma 2 (PLCg2), a direct downstream target of BTK, were identified in CLL patients who developed secondary resistance to ibrutinib. ${ }^{59}$

-Less is known about resistance mechanisms to PI3Kס inhibitors. In MCL, high expression of PI3Ka has been associated with resistance to idelalisib. ${ }^{60}$ Whether overexpression of PI3Ka could play a similar role in CLL is not clear.

-Patients who failed to respond to the SYK inhibitor fostamatinib had much higher CD38 expression than responding patients. ${ }^{55}$

Consistent with the appearance that the mechanism of resistance differs between different BCR inhibitors, switching from one inhibitor to another can be effective. ${ }^{57}$ Patients progressing with disease transformation on ibrutinib have been reported to have a median survival of less than four months. ${ }^{58}$ The poor outcome for these patients may reflect the adverse biological characteristics of their particular disease and, at least for patients in the early studies, the absence of effective alternative agents. With the availability of different kinase inhibitors, novel agents such as the BCL-2 antagonist ABT-199, or more effective immunotherapies CAR $T$ cells or stem cell transplantation, the outlook for these patients is expected to improve. ${ }^{61}$

As we have seen that targeted therapy resistance presents a clinical challenge, it would certainly be preferable to prevent resistance from developing by using a 
combination protocol along with other chemotherapy or immunochemotherapy or using more than one target therapy in same protocol. .

What is the role of Venetoclax in CLL?

Venetoclax is an orally bioavailable, selective BCL-2 antagonist ABT-199. It is a $\mathrm{BH} 3$ mimetic which acts similarly to the $\mathrm{BH} 3$-only proteins which antagonize $\mathrm{BCL} 2$ and its prosurvival family members. ${ }^{62}$ It is not currently FDA approved, but is being tested in late-stage clinical studies in CLL. In phase 1 study with a median followup of 15 months, the ORR was $77 \%$, with a high (CR) rate of $23 \%$. Estimated median PFS is 18 months. ${ }^{63}$ Incombination with rituximab, at a median follow-up of about 7 months, the ORR was $88 \%$, with 6 of 49 patients experiencing disease progression. This drug has been shown in clinical studies to induce minimal residual disease-negative $\mathrm{CRs}$, which are generally not seen withPI3K and BTK inhibitors. ${ }^{64}$

Is there a role for lenalidomide in CLL?

Lenalidomide is an immunomodulatory drug that was approved for multiple myeloma and myelodysplastic syndrome .It shows clinical activity in patients with CLL under clinical trial but it is not yet FDA approved.

Strati et al., investigated the activity of lenalidomide as frontline treatment of older patients with $\mathrm{CLL}^{65}$, they evaluated long-term outcomes of 60 patients with CLL treated with an initial therapy of lenalidomide. At a median follow-up of 4 years, time-to-treatment failure has not been reached and overall survival is $82 \%$. Thirtyfive $(58 \%)$ patients had a response lasting $>36$ months (long-term responders [LTRs]). Best LTR responses consisted of $25(71 \%)$ CR and $10(29 \%)$ PR. In addition to clinical responses, an increase in immunoglobulin levels of $>50 \%$ from baseline was reported. Normalization in the percentage of CD4 and CD8 cells and T-cell numbers was also observed.

Combination trials with rituximab showed promising results for relapsed patients or as initial therapy. The overall response rate was $66 \%$, including $12 \% \mathrm{CR}$ and $12 \%$ nodular PR. Time to treatment failure was 17.4 months. Median overall survival has not been reached; estimated survival at 36 months is $71 \%$. The most common grade 3 or 4 toxicity was neutropenia (73\% of patients). ${ }^{66}$ Lenalidomide treatment frequently induces an acute inflammatory reaction in CLL patients, the so-called "tumor flare reaction" that is suggestive of an immune activation phenomenon. Therefore, it seems more likely that the efficacy of lenalidomide in CLL results from both an antiproliferative activity on the malignant cells and an immunomodulatory effect on their microenvironment. ${ }^{67}$

Is still a role for HSCT in CLL therapy in new era?

Allogeneic hematopoietic stem cell transplantation (HSCT) has been regarded as treatment of choice for patients has refractory disease to the standard treatment or progresses after a short period of time if they are eligible for transplantation. In 2007, a consensus paper identified high-risk CLL (HR-CLL; disease refractory to purine analogs, disease relapsing within 2 years after purine analog combination treatment, and/or disease with del[17p]/TP53 mutations) as a situation in which HSCT should be considered. 68

The basis for HSCT in CLL is graft-versus-leukemia (GVL) activity. Evidence for GVL efficacy in CLL derives from the lower relapse risk after chronic graft-versushost disease (GVHD) ${ }^{69}$ MRD negativity can be reached in up to $50 \%$ of patients allografted for HR-CLL, suggesting that HSCT is capable of curing the disease. ${ }^{70} \mathrm{HSCT}$ overcomes the poor prognostic impact of genetic risk factors and fludarabine refractoriness. ${ }^{69}$ 
Prognosis of patients with HR-CLL who relapse or progress after HSCT appears to be not necessarily poorer than that of patients with HR-CLL who have not received a transplant. ${ }^{71}$

Modern HSCT approaches as reduced intensity conditioning (RIC) HSCT strategies are much less toxic than traditional myeloablative conditioning regimens. The early death rate of CLL post transplants (i.e., death within the first 100 days after HSCT) has dramatically decreased from previous rates of up to $40 \%$ with traditional conditioning to less than $5 \%$ in the most recent studies with RIC, but the main morbidity is due to GVHD and its complication. ${ }^{72}$

The established treatment algorithm for CLL are currently challenged by novel classes of drugs such BCR signal inhibitors and the selective BCL2 antagonist. The reported response rates in patients with relapsed/refractory $(R / R) \quad C L L$ (including $\mathrm{HR}-\mathrm{CLL}$ ) treated with $\mathrm{BCRi} / \mathrm{BCL} 2 \mathrm{a}$ are uniformly good. ${ }^{51}$ Despite the excellent ORR, complete remissions are seen in only a minority of patients treated with BCRi/BCL2a ${ }^{73}$ The long-term efficacy of novel agents in CLL treatment is largely unknown. There is no information about long-term toxicity and optimum duration of treatment.

So every patient with HR-CLL who is indicated for treatment should be offered one of the new drugs to induce disease control, ideally within a clinical trial. Once maximum response is achieved, there are 2 options: perform a consolidating HSCT or continue on BCRi/BCL2a until progression, thereby postponing the HSCT option to the next treatment line. It is important however, that HSCT is not considered a last resort treatment, offered to patients only after all other options have been exhausted. ${ }^{74,75}$

CAR-modified T cells have been developed to target CD19 on CLL and other malignancies.CD19 is an excellent tumor target; it is expressed throughout B-cell development, is expressed on almost all B-cell malignancies. The use of autologous T cells to target CLL has several potential advantages; there is no risk for GVHD, long-term immunosuppression is not needed, and autologous cells can survive for long periods of time and provide ongoing protection against relapse. Bcell aplasia and Cytokine release syndrome are considered its main toxicity. it shows a promising result in CLL clinical trials. ${ }^{74}$

After new revolution in CLL therapy how can you treat refractory/relapsed CLL?

Treatment options for relapsed or refractory CLL have markedly improved in recent decades; however there is no standard approach has been validated in clinical trial till now.

The choice of therapy after relapse depends on a number of individual factors as age, comorbidities of the patient, clinical parameters such as the type of primary treatment and the duration of remission achieved.

-Patients who fail to respond to current standard therapies (FCR, BenR, ClbR ,ClbObi, ClbOfa, BenOfa) or who undergo an early relapse (<2 years), or relapsed patients with a confirmed del(17p13) or a TP53 mutation have a poor prognosis. Their median overall survival time is just 1 - 2 years from the date of the initiation of salvage therapy. Choice of therapy for those -patients depends on their fitness. ${ }^{76}$ If patient is physically fit and eligible for HSCT you should advise him to go for HSCT as first option.${ }^{74}$ and you can give him option of one of the novel therapy as ibrutinib, idelalisib or BCL2 inhibitor. ${ }^{45,51,63}$

If patient is physically unfit and has no access to novel therapy you can try alemtuzumab or another less aggressive combination therapy. ${ }^{34}$ 
-Patients with late relapse (Late relapse who relapse 2-3 years from initiation on first line), it seems justified in principle, where there is a good response and a longer duration of remission of at least 2-3 years depending on the intensity of the therapy, to use the same regimen again as was used for the first-line therapy, other available relapse therapies include one of novel therapies as ibrutinib, idelalisib or other combination chemoimmunotherapy. ${ }^{45,51}$

\section{Conclusion:-}

Chronic lymphocytic leukemia is a heterogeneous disease with variable clinical course that has become more predictable with better understanding of disease biology and newer prognostic factors . Traditional chemotherapy is associated with acute and long term toxicity and is considered undesirable by most patients. In recent years, interest in non-chemotherapeutic approaches has been stimulated by an increasing number of therapeutic agents that target cell surface, intracellular pathways, and the tumor microenvironment. The challenge will be to develop scientifically rational combinations of these agents that will probably vary on a disease and even patient basis. Despite these improvements, the disease remains incurable, and much work still remains to ensure that we move toward a cure in this disease.

\section{References:}

1. Elter $T$, Hallek $M$, Engert $A$. Fludarabine in chronic lymphocytic leukaemia. Expert OpinPharmacother. 2006 Aug. 7(12):1641-51.

2. Cancer Facts \& Figures 2016. American Cancer Society. Available athttp://www.cancer.org/acs/groups/content/@research/documents/doc ument/acspc-047079.pdf. Accessed: March 10, 2016.

3. PDQ Adult Treatment Editorial Board. Chronic Lymphocytic Leukemia Treatment (PDQ®): Health Professional Version. PDQ Cancer Information Summaries. Bethesda, MD: National Cancer Institute; January 29, 2016.

4. Slager SL, Kay NE. Familial Chronic Lymphocytic Leukemia: What Does it Mean to Me?. Clin Lymphoma Myeloma. 2009 Sep 1. 9:S194-S197.

5. Berndt SI, Camp NJ, Skibola CF, Vijai J, Wang Z, et al. Meta-analysis of genome-wide association studies discovers multiple loci for chronic lymphocytic leukemia. Nat Commun. 2016 Mar 9. 7:10933.

6. Calin GA, Dumitru CD, Shimizu M, et al. Frequent deletions and downregulation of micro- RNA genes miR15 and miR16 at $13 q 14$ in chronic lymphocytic leukemia. ProcNatlAcadSciU S A. 2002;99(24):1552415529.

7. Zenz T, Benner A, Duhrsen U, Durig J, Dohner H, Siffert W, et al. BCL2$938 \mathrm{C}>\mathrm{A}$ polymorphism and disease progression in chronic lymphocytic leukemia. Leuk Lymphoma. 2009 Sep 11. 1-6. 
8. Hanlon K, Rudin CE, Harries LW. Investigating the targets of MIR-15a and MIR-16-1 in patients with chronic lymphocytic leukemia (CLL). PLoS One. 2009 Sep 25. 4(9):e7169.

9. Fabbri M, Bottoni A, Shimizu M, et al. Association of a microRNA/TP53 feedback circuitry with pathogenesis and outcome of B-cell chronic lymphocytic leukemia. JAMA. 2011 Jan 5. 305(1):59-67.

10. Zenz T, Mertens D, Kuppers R, Dohner H, Stilgenbauer S. From pathogenesis to treatment of chronic lymphocytic leukaemia.Nat Rev Cancer. 2010;10(1):37-50.

11. Puente XS, Pinyol M, Quesada V, et al. Whole-genome sequencing dentifies recurrent mutations in chronic lymphocytic leukaemia. Nature. 2011;475(7354):101-105.

12. Burger JA, Ghia P, Rosenwald A, Caligaris-Cappio F. The microenvironment in mature B-cell malignancies: a target for new treatment strategies. Blood. 2009;114(16):3367-3375

13. Stevenson FK, Krysov S, Davies AJ, Steele AJ, Packham G. B-cell receptor signaling in chronic lymphocytic leukemia. Blood. 2011;118(16):4313-4320.

14. Cramer $P$, HallekM. Prognostic factors in chronic lymphocytic leukemiawhat do we need to know? Nat Rev ClinOncol. 2011;8(1):38-47.

15. Hamblin TJ, Davis Z, Gardiner A, et al. UnmutatedlgV $(\mathrm{H})$ genes are associated with a more aggressive form of chronic lymphocytic leukemia. Blood. 1999;94(6):1848-1854.

16. Kröber A, Bloehdorn J, Hafner $S$, et al. Additional genetic high-risk features such as $11 q$ deletion, $17 p$ deletion, and V3-21 usage characterize discordance of ZAP-70 and $\mathrm{VH}$ mutation status in chronic lymphocytic leukemia. J ClinOncol. 2006;24(6):969-975.

17. National Center for Biotechnology Information USNLoM. CD38 CD38 molecule [Homo sapiens]. http://www.ncbi.nlm.nih.gov/pubmed?Db=gene\&Cmd=retrieve\&dopt=full _report\&list_uids=952. Accessed May 24, 2011

18. Deaglio S, Aydin S, Grand MM, et al. CD38/CD31 interactions activate genetic pathways leading to proliferation and migration in chronic lymphocytic leukemia cells. Mol Med. 2010;16(3-4):87-91.

19. Dohner H, Stilgenbauer S, Benner A, et al. Genomic aberrations and survival in chronic lymphocytic leukemia. $N$ Engl $J$ Med. 2000;343(26):1910-1916.

20. Hallek M, Cheson BD, Catovsky D, et al. Guidelines for the diagnosis and treatment of chronic lymphocytic leukemia: a report from the International Workshop on Chronic Lymphocytic Leukemia updating the National Cancer Institute-Working Group 1996 guidelines. Blood. 2008 Jun 15. 111(12):5446-56

21. Chemotherapeutic options in chronic lymphocytic leukemia: a metaanalysis of the randomized trials:CLLTrialists' Collaborative Group. J Natl Cancer Inst. 1999;91(10):861-868. 
22. Gribben JG. One step back but 2 steps forward. Blood. 2009 Oct 15 ;114(16):3359.

23. Moreton $\mathrm{P}$, Kennedy $\mathrm{B}$, Lucas $\mathrm{G}$, et al. Eradication of minimal residual disease in B-cell chronic lymphocytic leukemia after alemtuzumab therapy is associated with prolonged survival. J Clin Oncol.2005;23:29712979.

24. Hallek M, Fischer K, Fingerle-Rowson G, Fink AM, Busch R, Mayer J, et al. Addition of rituximab to fludarabine and cyclophosphamide in patients with chronic lymphocytic leukaemia: a randomised, open-label, phase 3 trial. Lancet 2010 Oct 2;376(9747):1164-1174

25. Eichhorst BF, Busch R, Hopfinger G, Pasold R, Hensel M, Steinbrecher $\mathrm{C}$, et al. Fludarabine plus cyclophosphamide versus fludarabine alone in first-line therapy of younger patients with chronic lymphocytic leukemia. Blood 2006 Feb 1;107(3):885-891

26. Eichhorst E, Fink A, Busch R, Lange $E$, Koppler $H$, Kiehl $M$, et al. Chemoimmunotherapy with Fludarabine $(F)$, Cyclophosphamide $(C)$, and Rituximab (R) (FCR) versus Bendamustine and Rituximab (BR) In Previously Untreated and Physically Fit Patients (pts) with Advanced Chronic Lymphocytic Leukemia (CLL): Results of a Planned Interim Analysis of the CLL 10 Trial, An International Randomized Study of the German CLL Study Group (GCLLSG). 55th ASH Annual Meeting and Exposition Abstract 2014; Oral Session 642.

27. . Goede V, Fischer K, Busch R, Engelke A, Eichhorst B, Wendtner CM, et al. Obinutuzumab plus chlorambucil in patients with CLL and coexisting conditions. N Engl J Med 2014 Mar 20;370(12):1101-1110

28. Voso MT, Pantel G, Rutella S, et al. Rituximab reduces the number of peripheral blood B-cells in vitro mainly by effector cell-mediated mechanisms. Haematologica. 2002;87:918-925.

29. O'Brien SM, Kantarjian H, Thomas DA, et al. Rituximab dose-escalation trial in chronic lymphocytic leukemia. J ClinOncol. 2001;19:2165-2170.

30. Wierda WG, Kipps TJ, Mayer J, et al. Ofatumumab as single-agent CD20 immunotherapy in fludarabine-refractory chronic lymphocytic leukemia. J ClinOncol. 2010;28(10).

31. Patz M, Isaeva P, Forcob N, et al. Comparison of the in vitro effects of the anti-CD20 antibodies rituximab and GA101 on chronic lymphocytic leukaemia cells. Br J Haematol. 2011;152(3):295-306.

32. Mossner E, Brunker P, Moser S, et al. Increasing the efficacy of CD20 antibody therapy through the engineering of a new type II anti-CD20 antibody with enhanced direct and immune effector cell-mediated B-cell cytotoxicity. Blood. 2010;115(22):4393-4402.

33. Kaufman M, Rai KR. Alemtuzumab in the up-front setting. TherClin Risk Manag 2008 Apr;4(2):459-464

34. Stilgenbauer $\mathrm{S}$, Dohner $\mathrm{H}$. Campath-1H-induced complete remission of chronic lymphocytic leukemia despite p53 gene mutation and resistance to chemotherapy. N Engl J Med 2002 Aug 8;347(6):452-453

35. Pettitt AR, Jackson R, Carruthers S, Dodd J, Dodd S, Oates M, et al. Alemtuzumab in combination with methylprednisolone is a highly effective induction regimen for patients with chronic lymphocytic leukemia and deletion of TP53: final results of the national cancer research institute CLL206 trial. J ClinOncol 2012 May 10;30(14):1647-1655. 
36. Elter T, Gercheva-Kyuchukova L, Pylylpenko H, Robak T, Jaksic B, Rekhtman G, et al. Fludarabine plus alemtuzumab versus fludarabine alone in patients with previously treated chronic lymphocytic leukaemia: a randomised phase 3 trial. Lancet Oncol 2011 Dec;12(13):1204-1213.

37. Woyach JA, Johnson AJ, Byrd JC. The B-cell receptor signaling pathway as a therapeutic target in CLL. Blood. 2012;120(6):1175-1184.

38. Herishanu Y, P'erez-Gal'an P, Liu D, et al. The lymph node microenvironment promotes B-cell receptor signaling, NF-kappaB activation, and tumor proliferation in chronic lymphocytic leukemia. Blood. 2011;117(2):563-574.

39. Herman SE, Gordon AL, Wagner AJ, etal.Phosphatidylinositol 3-kinased inhibitor CAL-101 shows promising preclinical activity in chronic lymphocytic leukemia by antagonizing intrinsic and extrinsic cellular survival signals. Blood. 2010;116(12):2078-2088.

40. Buggy JJ, Elias L. Bruton Tyrosine Kinase (BTK) and Its Role in B-cell Malignancy. Int Rev Immunol. 2012;31(2):119-132.

41. O'Brien S, Furman RR, Coutre SE, et al. Ibrutinib as initial therapy for elderly patients with chronic lymphocytic leukaemia or small lymphocytic lymphoma:an open-label, multicentre, phase 1b/2 trial.LancetOncol. 2014;15(1):48-58.

42. Herman SE, Niemann $C U$, Farooqui $M$ et al. Ibrutinibinduced lymphocytosis in patients with chronic lymphocytic leukemia: correlative analyses from a phase II study. Leukemia. 2014 Nov;28(11):2188-96

43. Byrd JC, Brown JR, O'Brien S, et al. Ibrutinib versus Ofatumumab in Previously Treated Chronic Lymphoid Leukemia. $\mathrm{N}$ Engl J Med. 2014;371(3):213-223.

44. Farooqui MZ, Valdez J, Martyr S, et al.Ibrutinib for previously untreated and relapsed or refractory chronic lymphocytic leukaemia with TP53 aberrations: a phase 2,single-arm trial. Lancet Oncol. 2015;16 (2):169176.

45. Burger JA, Keating MJ, Wierda WG, et al. Safety and activity of ibrutinib plus rituximab for patients with high-risk chronic lymphocytic leukaemia: a single-arm, phase 2 study. Lancet Oncol. 2014;15(10):1090-1099.

46. Jaglowski SM, Jones JA, Nagar V, etal.Safety and activity of BTK inhibitor ibrutinib combined with ofatumumab in chronic lymphocytic leukemia: a phase 1b/2 study.Blood. 2015;126(7):842-850.

47. Brown JR, Barrientos JC, Barr PM, et al. The Bruton tyrosine kinase inhibitor ibrutinib with chemoimmunotherapy in patients with chronic lymphocytic leukemia. Blood.2015;125(19):2915-2922.

48. Herman SE, Johnson AJ. Molecular pathways: targeting phosphoinositide 3-kinase p110-delta in chronic lymphocytic leukemia.Clin Cancer Res. 2012;18(16):4013-4018.

49. Chantry D, Vojtek A, Kashishian A, et al. p110delta, a novel phosphatidylinositol 3-kinase catalytic subunit that associates with p85 and is expressed predominantly in leukocytes. $J$ Biol Chem.1997;272(31):19236-19241. 
50. So L, Fruman DA. PI3K signalling in B- and T-lymphocytes: new developments and therapeutic advances. Biochem J. 2012;442 (3):465481.

51. Furman RR, Sharman JP, Coutre SE, etal.Idelalisib and rituximab in relapsed chronic lymphocytic leukemia. N Engl J Med. 2014;370(11):9971007.

52. O'Brien SM, Lamanna N, Kipps T et al.A phase 2 study of idelalisib plus rituximab in treatment-naïve older patients with chronic lymphocytic leukemia.blood. 2015 Dec 17;126(25):2686-94

53. Coutre SE, Barrientos JC, Brown JR, etal.Management of adverse events associated with idelalisib treatment: expert panel opinion.Leuk Lymphoma. 2015;1-8.

54. Louie CY, DiMaio MA, MatsukumaKE,Coutre SE, Berry GJ, Longacre TA. IdelalisibassociatedEnterocolitis: Clinicopathologic Features and Distinction From Other Enterocolitides. Am J SurgPathol. 2015 Sept 29.

55. Friedberg JW, Sharman J, Sweetenham J, et al. Inhibition of Syk with fostamatinib disodium has significant clinical activity in non-Hodgkin lymphoma and chronic lymphocytic leukemia. Blood. 2010;115(13):25782585.

56. Hendriks RW, Yuvaraj S, Kil LP. Targeting Bruton's tyrosine kinase in B cell malignancies. Nat Rev Cancer. 2014;14(4):219-232.

57. Byrd JC, Furman RR, Coutre SE, et al. Threeyear follow-up of treatmentnaive and previously treated patients with CLL and SLL receiving singleagent ibrutinib. Blood.2015;125(16):2497-2506.

58. Maddocks KJ, Ruppert AS, Lozanski G, et al. Etiology of IbrutinibTherapyDiscontinuation and Outcomes in Patients WithChroniclymphocytic Leukemia. JAMA Oncol. 2015;1(1):80-87.

59. Woyach JA, Furman RR, Liu TM, etal.Resistance mechanisms for the Bruton's tyrosine kinase inhibitor ibrutinib. $\mathrm{N}$ EnglJMed. 2014;370(24):2286-2294.

60. lyengar S, Clear A, Bodor C, et al. P110alpha-mediated constitutive PI3K signaling limits the efficacy of p110delta-selective inhibition in mantle cell lymphoma, particularly with multiple relapse. Blood. 2013;121(12):22742284.

61. Woyach JA and Johnson AJ. Targeted therapies in CLL: mechanisms of resistance and strategies for management. BLOOD, 2015 :126 ( 4):471:477.

62. Anderson MA, Huang D, Roberts A. Targeting BCL2 for the treatment of lymphoid malignancies. SeminHematol. 2014;51(3):219-227.

63. Seymour JF, Davids MS, Pagel JM, et al. ABT- 199 (GDC-0199) in Relapsed/refractory (R/R) chronic lymphocytic leukemia (CLL) and small lymphocytic lymphoma (SLL): high complete response rate and durable disease control [abstract]. J ClinOncol. 2014;32(suppl 5s).Abstract 7015.

64. Roberts AW, Ma S, Brander DM, etal.Determination of recommended phase 2 dose of ABT-199 (GDC-0199) combined with rituximab (R) in patients with relapsed/refractory $(R / R)$ chronic lymphocytic leukemia (CLL) [abstract].Blood. 2014;124(21). Abstract 325. 
65. Strati $P$, Keating MJ, Wierda WG et al. Lenalidomide induces long-lasting responses in elderly patients with chronic lymphocytic leukemia. BLOOD, 2013, 122( 5):734.

66. Badoux XC etalPhase II study of lenalidomide and rituximab as salvage therapy for patients with relapsed or refractory chronic lymphocytic leukemia. J ClinOncol. 2013 Feb 10;31(5):584-91

67. Giannopoulos K, Mertens D, Stilgenbauer S. Treating chronic lymphocytic leukemia with thalidomide and lenalidomide. Expert OpinPharmacother. 2011;12(18):2857-2864.

68. Dreger P, Corradini P, Kimby E, et al; Chronic Leukemia Working Party of the EBMT. Indications for allogeneic stem cell transplantation in chronic lymphocytic leukemia: the EBMT transplant consensus. Leukemia. 2007;21(1):12-17.

69. Hahn M, B" ottcher S, Ritgen M, et al. Timing of immunosuppression tapering, chronic GVHD, and minimal residual disease (MRD) eradication in patients allografted for poor-risk chronic lymphocytic leukemia (CLL) as defined by the EBMT consensus criteria: A single center experience [abstract]. Bone Marrow Transplant.2013;48(S2):S72.

70. Bottcher S, Ritgen M, Dreger P. Allogeneic stem cell transplantation for chronic lymphocytic leukemia: lessons to be learned from minimal residual disease studies. Blood Rev. 2011;25(2):91-96.

71. Benjamini $O$, Rozovski U, Jain $P$, et al. Outcome Of Chronic Lymphocytic Leukemia (CLL) Patients That Failed Allogeneic Stem Cell Transplantation.[abstract]. Blood. 2013;122(21).

72. Herth I, Dietrich S, Benner A, et al. The impact of allogeneic stem cell transplantation on the natural course of poor-risk chronic lymphocytic leukemia as defined by the EBMT consensus criteria:a retrospective donor versus no donor comparison. Ann Oncol. 2014;25(1):200-206.

73. Byrd JC, Furman RR, Coutre SE, et al. Targeting BTK with ibrutinib in relapsed chronic lymphocytic leukemia. N Engl J Med. 2013;369(1):3242.

74. Mato A and. Porter D. A drive through cellular therapy for CLL in 2015: allogeneic cell transplantation and CARs. BLOOD, $2015 ; 126,(4): 478$

75. Weisdorf D, Spellman S, Haagenson M, et al.Classification of HLAmatching for retrospective analysis of unrelated donor transplantation: revised definitions to predict survival. Biol Blood Marrow Transplant. 2008;14(7):748-758.

76. Dreger $\mathrm{P}$, Schetelig J, Andersen $\mathrm{N}$ et al: Managing high-risk chronic lymphocytic leukemia during transition to a new treatment era: Stem cell transplantation or novel agents. A position statement of the European Research Initiative on CLL (ERIC) and the European Society for Blood and Marrow Transplantation (EBMT). 